\title{
Comment on the choice of time in a two-component formulation of the Wheeler-DeWitt equation
}

\author{
Marc Thibeault* and Claudio Simeone ${ }^{\dagger}$ \\ Departamento de Física, Facultad de Ciencias Exactas y Naturales, \\ Universidad de Buenos Aires, Ciudad Universitaria Pab. I, \\ 1428, Buenos Aires, Argentina
}

September 18, 2018

\begin{abstract}
The two-component formalism in quantum cosmology is revisited with a particular emphasis on the identification of time. Its relation with the appearance of imaginary eigenvalues is established. It is explicitly shown how a good choice of the global time prevents this peculiarity.
\end{abstract}

KEY WORDS: Minisuperspace; Wheeler-DeWitt equation; global phase time.

PACS numbers: $04.60 . \mathrm{Kz} \quad$ 04.60.Gw $98.80 . \mathrm{Hw}$

In Ref. [1] an interesting proposal was introduced for solving the problem of the interpretation of the Wheeler-DeWitt equation [2, 3] for minisuperspaces. The proposal is inspired in Dirac's solution to the problems of the Klein-Gordon equation, and consists in writing the Wheeler-DeWitt equation as a two-component Schrödinger equation. This formulation is a possible way for associating a system of differential equations which are first order in the derivative respect to time to a Klein-Gordon type equation, as it is the Wheeler-DeWitt one. This allows to obtain an equation which admits well known resolution procedures and an interpretation in terms of a well defined inner product. In the program developed in Refs. [1, 4, 5] it was shown that such idea can be applied to minisuperspace models. The procedure reduces the resolution of the Wheeler-DeWitt equation to an eigenvalue problem and a series

*e-mail: marc@df.uba.ar

†e-mail: csimeone@df.uba.ar 
of algebraic equations which can be solved by iteration. The proposal has been developed within the framework of the quantization of Friedmann-Robertson-Walker (FRW) minisuperspaces. In the case of a closed $(k=1)$ model with matter in the form of a massive scalar field, eigenvalues which become imaginary as the system evolves were obtained in one of the steps of the application of the formalism. Though the program of Refs. [1, 4, 5] does not rely on a standard Schrödinger equation, where imaginary eigenvalues lead to a non unitary quantum description, the point deserves, we believe, a closer look which goes beyond the particular models considered in [1]. The possible relation existing between this peculiarity and the particular choice of time variable has been already pointed in Ref. [6]. The purpose of the present note is to carry out a detailed analysis of this issue within the context of the search for a consistent minisuperspace quantization with a globally right notion of time.

For a FRW minisuperspace with scaled constraint of the form

$$
H=\eta^{\mu \nu} p_{\mu} p_{\nu}+V(q)=0
$$

where $\eta^{\mu \nu}=\operatorname{diag}(-1,1)$ and $p$ are the momenta conjugated to the coordinates $q$, the program of Refs. [1, 4, 5] starts with the identification of $q^{0}$ as time variable and the definition of the operator

$$
\hat{D} \equiv-\frac{\partial^{2}}{\partial\left(q^{1}\right)^{2}}+\hat{V}(q)
$$

Then the second order equation resulting from the Hamiltonian constraint is reduced defining a two-component wave function

$$
\Psi=\frac{1}{\sqrt{2}}\left(\begin{array}{c}
\psi+i \dot{\psi} \\
\psi-i \dot{\psi}
\end{array}\right)
$$

where dots mean derivatives respect to $q^{0}$, and introducing the time-dependent Hamiltonian operator

$$
\hat{H}=\frac{1}{2}\left(\begin{array}{cc}
1+\hat{D} & -1+\hat{D} \\
1-\hat{D} & -1-\hat{D}
\end{array}\right)
$$

This leads to the Schrödinger equation

$$
i \dot{\Psi}=\hat{H} \Psi
$$

which is then solved by finding the solutions for the eigenvalue problem

$$
\hat{H} \Psi_{n}=E_{n} \Psi_{n} .
$$


With the definition

$$
\Psi_{n}=\frac{1}{\sqrt{2}}\left(\begin{array}{c}
1+E_{n} \\
1-E_{n}
\end{array}\right) \Phi_{n}
$$

one obtains

$$
\hat{D} \Phi_{n}=E_{n}^{2} \Phi_{n}
$$

In Ref. [1] the particular case of a model of arbitrary curvature with a massive scalar field $\phi$ was considered, with the choice $q^{0}=\Omega$, so that $\hat{D}=-\frac{\partial^{2}}{\partial \phi^{2}}-k e^{4 \Omega}+m^{2} \phi^{2} e^{6 \Omega}$. Thus Eq. (8) results to be

$$
\left\{-\frac{\partial^{2}}{\partial \phi^{2}}+m^{2} \phi^{2} e^{6 \Omega}\right\} \Phi_{n}=\mathcal{E}_{n} \Phi_{n}
$$

where $\mathcal{E}_{n}=E_{n}^{2}+k e^{4 \Omega}$ (see Ref. [4] for the details). In this case $\mathcal{E}_{n}$ are chosen as the energies of the harmonic oscillator. However, for the closed $(k=1)$ model, the eigenvalues $E_{n}=\sqrt{\mathcal{E}_{n}-e^{4 \Omega}}$ become imaginary as $\Omega$ increases: this happens for $e^{\Omega}>m$ (the corresponding eigenvectors are null).

Now, it is known that for the closed FRW model the logarithm of the scale factor is not a globally good time variable since its evolution is not monotonous (the model undergoes a re-collapse). A globally good time is a function $t\left(q^{i}, p_{i}\right)$ which monotonically increases along a dynamical trajectory, that is, each surface $t=$ constant in the phase space is crossed by a dynamical trajectory only once; hence the successive states of the system can be parametrized by this function. This means that $t\left(q^{i}, p_{i}\right)$ must fulfill the condition [7]

$$
[t, H]>0
$$

Because the general form of the Hamiltonian constraint is $H=G^{i k} p_{i} p_{k}+V(q)=0$ and the supermetric $G^{i k}[2,3]$ does not depend on the momenta, a function of only the coordinates $t\left(q^{i}\right)$ is a global time if the bracket $\left[t\left(q^{i}\right), G^{i k} p_{i} p_{k}\right]=2 G^{i k} p_{k} \partial t / \partial q^{i}$ is positive definite. Hence if the supermetric has a diagonal form and one of the momenta vanishes at a given point of phase space, then no function of only its conjugated coordinate can be a global time. For a constraint whose potential can be zero for finite values of the coordinates, the momenta $p_{k}$ can be all equal to zero at a given point, and $\left[t\left(q^{i}\right), H\right]$ can vanish. Hence, what is called an intrinsic time $t\left(q^{i}\right)$ [8] exists only if the potential in the constraint has a definite sign; this is closely related with the existence of a globally well defined canonical gauge condition $\chi(q, \tau)=0$ for the system [9], as it is to be expected from the fact that in the theory of gravitation the dynamical evolution can be reproduced by gauge transformations [3]. In the 
most general case a global phase time should be a function including the canonical momenta; this is called an extrinsic time $t\left(q^{i}, p_{i}\right)[10]$.

To show explicitly the relation of imaginary eigenvalues problem with a choice of time which is not globally right we shall consider a model which, despite its simplicity, allows for a detailed analysis of the central aspects of the problem. A suitable example is given by an isotropic and homogeneous dilaton cosmology described by the scaled Hamiltonian constraint

$$
H=-p_{\Omega}^{2}+p_{\phi}^{2}+2 c e^{6 \Omega+\phi}+\lambda^{2} e^{-2 \phi}=0,
$$

which corresponds to a flat universe with dilaton field $\phi$ and non vanishing antisymmetric field $B_{\mu \nu}$ coming from the $N S-N S$ sector of effective string theory; the existence of this field is reflected only in the positive definite constant $\lambda^{2}$ (see Refs. [11, 12]). Because we are interested in problems of the formalism only, we shall consider $c$ as an arbitrary constant. In the case $c<0$ the potential can vanish, so that the system does not admit an intrinsic time. In the low energy string theory one can consider the limit $\phi \rightarrow-\infty$, then the $e^{\phi} \equiv V(\phi)$ factor in the first term of the potential verifies $V(\phi)=V^{\prime}(\phi) \ll 1$, and we can replace $c e^{\phi}$ by the constant $\bar{c}$ fulfilling $|\bar{c}| \ll|c|$ :

$$
H=-p_{\Omega}^{2}+p_{\phi}^{2}+2 \bar{c} e^{6 \Omega}+\lambda^{2} e^{-2 \phi}=0 .
$$

Following Ref. [1], the two-component formulation could now be followed starting from this constraint, after identifying the logarithm of the scale factor, $\Omega$, as time parameter. We shall see that, as it was the case for the closed FRW model in that work, in the case $\bar{c}<0$ this choice leads to imaginary eigenvalues. For the constraint (12), the application of the formalism of Ref. [1] gives

$$
\hat{D}=-\frac{\partial^{2}}{\partial \phi^{2}}+2 \bar{c} e^{6 \Omega}+\lambda^{2} e^{-2 \phi}
$$

and the corresponding eigenvalue equation

$$
\left\{-\frac{\partial^{2}}{\partial \phi^{2}}+2 \bar{c} e^{6 \Omega}+\lambda^{2} e^{-2 \phi}\right\} \Phi_{n}=E_{n}^{2} \Phi_{n}
$$

Then defining $\mathcal{E}_{n} \equiv E_{n}^{2}-2 \bar{c} e^{6 \Omega}$ we obtain

$$
\left\{-\frac{\partial^{2}}{\partial \phi^{2}}+\lambda^{2} e^{-2 \phi}\right\} \Phi_{n}=\mathcal{E}_{n} \Phi_{n}
$$

We see that, though choosing, as before, $\mathcal{E}_{n} \in \mathbb{R}$, in the case $\bar{c}<0$ the eigenvalues $E_{n}=\sqrt{\mathcal{E}_{n}+2 \bar{c} e^{6 \Omega}}$ corresponding to the operator $\hat{H}$ (see Eq. ([6]) become imaginary for 
large enough values of the scale factor, as it happens in Ref. [1] in the case of the closed $(k=1)$ model. Note that this is in correspondence with the fact that the classical quantity from which the operator $\hat{D}$ comes is $p_{\phi}^{2}+2 \bar{c} e^{6 \Omega}+\lambda^{2} e^{-2 \phi}$, which is not positive definite. But the operator $\hat{D}$ is defined starting from the previous identification of the time variable. Thus the existence of imaginary eigenvalues for the operator $\hat{H}$, in this sense, reflects that the variable chosen as time is not globally monotonous: Indeed, it is easy to see that $[\Omega, H]$ vanishes for finite values of the canonical variables (the same happens with the dilaton $\phi$ ): $[\Omega, H] \sim-p_{\Omega}$, and $p_{\Omega}$ passes continuously from positive to negative values, so that the scale factor stops growing and begins to decrease. Then there would exist an infinite range of values of "time" which are never reached; this range depends on the values of the other variables, i.e. $\phi$ and $p_{\phi}$, which is in correspondence with the fact that the range of values of $\Omega$ such that the eigenvalues become imaginary also depends on the dilaton.

In a description with a globally right notion of time, for $\bar{c}<0$ the scale factor $\Omega$ cannot be taken as the clock for the system. A right choice becomes apparent by performing the canonical transformation first introduced for the Taub universe in Ref. [13] in order to obtain a constraint with only one term in the potential. This is achieved by introducing the generating function of the first kind $f_{1}(\phi, s)= \pm|\lambda| e^{-\phi} \sinh s$. The new canonical variables are then given by $s= \pm \operatorname{arcsinh}\left(p_{\phi} e^{\phi} /|\lambda|\right), p_{s}= \pm|\lambda| e^{-\phi} \cosh s$. With this canonical transformation the resulting form for the Hamiltonian constraint in the limit $V(\phi)=V^{\prime}(\phi) \ll 1$ is 14

$$
H=-p_{\Omega}^{2}+p_{s}^{2}+2 \bar{c} e^{6 \Omega}=0,
$$

and we can apply the two-component formulation starting from this constraint. The central difference with a straightforward application of the formalism to the original constraint is that now the constraint surface splits into two disjoint sheets, identified by the sign of the momentum which does not vanish. The formalism thus includes two disjoint theories for the physical degree of freedom evolving with a globally right time. If $\bar{c}>0$, we have $p_{\Omega} \neq 0$ and $\Omega$ is a global time. But in the case $\bar{c}<0$, the momentum which does not vanish is $p_{s}$ and the time is $t= \pm s$. According to the definition of the new variable $s$, the time $t= \pm s$ is a function of both $p_{\phi}$ and $\phi$, then being effectively an extrinsic time. The application of the two-component formalism starting from the constraint (16) leads to different eigenvalue equations depending on the sign of $\bar{c}$. In the case $\bar{c}>0$ (which in fact does not require the 
canonical transformation), the global time is the scale factor, and we would obtain

$$
\left\{-\frac{\partial^{2}}{\partial s^{2}}\right\} \Phi_{n}=E_{n}^{2} \Phi_{n}
$$

that is, the eigenvalue equation $\hat{D} \Phi_{n}=\mathcal{E}_{n} \Phi_{n}$ with the time-dependent eigenvalues $E_{n}=$ $\sqrt{\mathcal{E}_{n}+2 \bar{c} e^{6 \Omega}}$, which are kept real as the system evolves. In the case $\bar{c}<0$ requiring an extrinsic time, instead, the eigenvalue equation $\hat{D} \Phi_{n}=\mathcal{E}_{n} \Phi_{n}$ turns to be

$$
\left\{-\frac{\partial^{2}}{\partial \Omega^{2}}-2 \bar{c} e^{6 \Omega}\right\} \Phi_{n}=E_{n}^{2} \Phi_{n},
$$

with $\mathcal{E}_{n}=E_{n}^{2}$, so that now the eigenvalues associated to the operator $\hat{H}$ are real in a trivial sense, as they do not change when the system evolves. In this case, differing from the situation with $\bar{c}<0$ before the canonical transformation, the operator $\hat{D}$ is the quantum version of the classical quantity $p_{\Omega}^{2}-2 \bar{c} e^{6 \Omega}$, which for $\bar{c}<0$ is positive definite; thus no imaginary eigenvalues would be expected to appear. We emphasize this point: when a right time is identified as a previous step before applying the quantization formalism, the operator $\hat{D}$ is associated to a classical quantity which cannot be negative nor zero for finite values of the canonical variables.

To obtain a better insight it can be helpful a comparison with the usual canonical quantization formalism, that is with the Wheeler-DeWitt or the Schrödinger formulations. As early pointed in Ref. [15], both formulations are equivalent when the time does not appear in the potential. For the models considered (see (12)), the problem of time which we have addressed appears in the case $\bar{c}<0$, for which, after the canonical transformation introduced above, one obtains a time-independent potential. Thus in this case both the Wheeler-deWitt and the Schrödinger formulations are equivalent (in the strong sense that the solutions are exactly the same). Choosing the Wheeler-deWitt equation we obtain:

$$
\left[-\frac{\partial^{2}}{\partial \Omega^{2}}+\frac{\partial^{2}}{\partial s^{2}}-2 \bar{c} e^{6 \Omega}\right] \Psi=0
$$

In terms of these new variables, the equation is separable. Proposing the ansatz $\Psi(\Omega, s)=$ $\Theta(\Omega) S(s)$ we obtain the two ordinary differential equations

$$
\begin{aligned}
\frac{d^{2} \Theta(\Omega)}{d \Omega^{2}}+2 \bar{c} e^{6 \Omega} \Theta(\Omega)-\kappa \Theta(\Omega) & =0 \\
\frac{d^{2} S(s)}{d s^{2}}-\kappa S(s) & =0
\end{aligned}
$$


where $\kappa$ is a separation constant. Unitarity of the evolution leads to the choice $\kappa=-E^{2}$. Then $S(s) \sim e^{i E s}$ and we recover Eq. (18) for the spatial part of the wave function

$$
\frac{d^{2} \Theta(\Omega)}{d \Omega^{2}}+2 \bar{c} e^{6 \Omega} \Theta(\Omega)+E^{2} \Theta(\Omega)=0 .
$$

Note that the spatial part of the Wheeler-DeWitt wave function coincides with the function $\Phi$ in this particular case but this is not a general feature. For example, this will not be true for $\bar{c}>0$. In this case a globally good time is $\Omega$ and the formalism of Ref. [1] presents no eigenvalue problems. However, the usual canonical quantization procedure itself is not so easy to interpret. In this case, as a result of the time-dependent potential, the WheelerDeWitt formalism is not equivalent to the Schrödinger formalism, thus making not trivial an interpretation in terms of conserved probabilities. In the Schrödinger picture the wave function for the physical degrees of freedom, say $x$, at a time $t$ is given by evolving the solution at an initial time $t_{0}$, which results from the equation [15, 6]:

$$
\hat{h}^{2}\left(x, t_{0}\right) \Psi_{E}\left(x, t_{0}\right)=E^{2} \Psi_{E}\left(x, t_{0}\right) .
$$

The eigenvalue equation $\hat{D} \Phi_{n}=E_{n}^{2} \Phi_{n}$ appears similar to this equation; in fact, the operator $\hat{h}^{2}$ has the same form of the operator $\hat{D}$. But while in the Schrödinger case the eigenvalues are constant -the corresponding eigenvalue equation (23) is solved at a fixed time $t_{0}$ - and the evolution is straightforwardly obtained by acting on $\Psi_{E}\left(x, t_{0}\right)$ with the evolution operator given by the integral of the time-dependent true Hamiltonian, in the two-component formulation time-dependent eigenvalues appear, and the evolution is not so immediately obtained.

We have then focused on a specific problem that arises in the two-component formalism introduced in Ref. [1]: the appearance of imaginary eigenvalues. As pointed in the cited work, this leads to a decomposition of the Hilbert space into three parts: negative norm vectors, positive norm vectors, and null vectors, being the latter associated to imaginary eigenvalues; this decomposition has the peculiarity of being variable, according to the evolution of the corresponding eigenvalues themselves. We have shown by means of a specific simple example that this feature can be seen to arise from a choice of time which is not globally right. In other words, we could say that the old but fundamental problem of time in quantum cosmology appears in another disguise. However, we have also showed within an analysis which could, in principle, be extended beyond the particular models considered here, that a good choice of time renders the proposed formalism free of the peculiarity of eigenvalues which evolve to become imaginary. 


\section{References}

[1] A. Mostafazadeh, J. Math. Phys. 39, 4499 (1998).

[2] J. J. Halliwell, in Introductory Lectures on Quantum Cosmology, Proceedings of the Jerusalem Winter School on Quantum Cosmology and Baby Universes, edited by T. Piran, World Scientific, Singapore (1990); J. J. Halliwell, in Cambridge 2002, The future of theoretical physics and cosmology, 675-692 (gr-qc/0208018).

[3] A. O. Barvinsky, Phys. Rep. 230, 237 (1993).

[4] A. Mostafazadeh, gr-qc/0205049.

[5] A. Mostafazadeh, Class. Quant. Grav. 20, 155 (2003).

[6] T. P. Shestakova and C. Simeone, Grav. Cosmol. 10, 161 (2004); gr-qc/0409114.

[7] P. Hájícek, Phys. Rev. D 34, 1040 (1986).

[8] K. V. Kuchař, in: Proceedings of the 4th Canadian Conference on General Relativity and Relativistic Astrophysics, ed. G. Kunstatter, D. Vincent and J. Williams, World Scientific, Singapore (1992).

[9] C. Simeone, J. Math. Phys. 40, 4527 (1999); C. Simeone, Deparametrization and Path Integral Quantization of Cosmological Models, World Scientific Lecture Notes in Physics 69, World Scientific, Singapore (2001); T. P. Shestakova and C. Simeone, Grav. Cosmol. 10, 257 (2004); gr-qc/0409119.

[10] K. V. Kuchař, Phys. Rev. D 4, 955 (1971); J. W. York, Phys. Rev. Lett. 28, 1082 (1972).

[11] M. Gasperini, in Proceedings of the 2nd SIGRAV School on Gravitational Waves in Astrophysics, Cosmology and String Theory, Villa Olmo, Como, edited by V. Gorini; hep-th/9907067.

[12] M. Gasperini and G. Veneziano, Phys. Rept. 373, 1 (2003).

[13] G. Catren and R. Ferraro, Phys. Rev. D 63, 023502 (2001).

[14] C. Simeone, Phys. Lett. A 310, 143 (2003).

[15] W. F. Blyth and C. J. Isham, Phys. Rev. D 11, 768 (1975). 